\title{
Validation of the Association of Genetic Variants on Chromosome 9p21 and 1q41 With Myocardial Infarction in a Japanese Population
}

\author{
Yumiko Hiura, PhD*; Yasue Fukushima, MS*; Miyuki Yuno*; Hiromi Sawamura, MS*; \\ Yoshihiro Kokubo, MD**; Tomonori Okamura, MD**; Hitonobu Tomoike, MD**; \\ Yoichi Goto, $\mathrm{MD}^{\dagger}$; Hiroshi Nonogi, $\mathrm{MD}^{\dagger}$; Rie Takahashi, PhD*; Naoharu Iwai, MD*,†
}

\begin{abstract}
Background Recent large-scale genome-wide association studies have identified several loci associated with the risk of coronary artery disease (CAD). The aim of the present study was to examine whether the previously reported CAD-associated single-nucleotide polymorphisms (SNPs) confer susceptibility to myocardial infarction (MI) in a study population of 2,475 controls and 589 cases of MI. The effect of the CAD-associated SNPs on cardiovascular risk factors in the control group was also investigated.

Methods and Results Significant associations were observed between 2 SNPs, rs1333049 on chromosome 9p21 and rs17465637 on chromosome 1q41, and MI, with odds ratios adjusted for age, sex, diabetes, hypertension and smoking habit of 1.47 (95\% confidence interval (CI), 1.15-1.89; corrected $\mathrm{p}=0.006)$ and $1.45(95 \% \mathrm{CI}$, 1.15-1.83; corrected $\mathrm{p}=0.006$ ) for rs 1333049 and rs 17465637 , respectively. None of the genotypes was associated with body mass index, plasma lipid profile, blood pressure, glucose, or hemoglobin A 1c. The genotypes also had no effect on the marker of inflammation (C-reactive protein) or atherosclerosis (mean and maximum carotid intima-media thickness).

Conclusions Although the underlying mechanisms are not clearly understood, the previously reported association between the 2 SNPs (rs1333049 and rs17465637) and MI was reproduced in this Japanese sample. (Circ J 2008; 72: 1213-1217)
\end{abstract}

Key Words: Genetics; Myocardial infarction; Polymorphism

B ased on recent large-scale genome-wide association studies, several loci associated with the risk of coronary artery disease (CAD) have been identified, ${ }^{1-3}$ among which the chromosome 9p21 has been the most consistent locus showing a strong association with CAD. A genome-wide scan conducted in Canadians (322 cases, 312 controls) and subsequent validation studies involving more than 2,326 cases and 10,427 controls found the genetic variations in the chromosome 9p21 region (rs10757274 and rs2383206) to be associated with CAD? Similarly, the genome wide association analysis in an Icelandic population demonstrated the association between myocardial infarction (MI) and the single-nucleotide polymorphisms (SNPs) on the chromosome 9p21 locus (rs1333040, rs2383207, and rs10116277)! Combined analysis of the initial Icelandic and 4 replication groups from Iceland and the United States revealed rs10757278 on chromosome 9p21 as the most significant SNP, with the population attributable risk for MI being estimated as $21 \%$ in general! In the analysis of the Welcome Trust Case Control Consortium (WTCCC) study?

(Received January 31, 2008; revised manuscript received March 26, 2008; accepted April 7, 2008)

*Department of Epidemiology, Research Institute, **Division of Preventive Cardiology and Division of Cardiology, National Cardiovascular Center, Suita, Japan

Mailing address: Naoharu Iwai, MD, Department of Epidemiology, Research Institute, National Cardiovascular Center, 5-7-1 Fujishirodai, Suita 565-8565, Japan. E-mail: iwai@ri.ncvc.go.jp

All rights are reserved to the Japanese Circulation Society. For permissions, please e-mail: cj@j-circ.or.jp genotyping was carried out in 1,926 CAD cases and 2,938 controls using the GeneChip Human Mapping 500K array (Affymetrix). Nine chromosomal regions were found to be significantly associated with CAD, 3 of which remained significant after testing for the association with $\mathrm{MI}$ in the German MI Family Study composed of 875 cases with MI and 1,644 controls, ${ }^{4}$ with the chromosome 9p21.3 locus showing the strongest association in both studies. Combined analysis of the WTCCC study and the German MI Family Study revealed an additional 4 loci associated with the increased risk of CAD.

Despite the different SNPs investigated from study to study, associations between the SNPs on chromosome 9p21 and the risk of CAD or MI have been consistently reported in case-control studies from different countries including South Korea, Italy,7 Germany, Sweden, and the United Kingdom6 The underlying mechanisms for the association between these susceptibility loci identified through genome-wide scanning and the risk of CAD/MI remain to be elucidated. There has been no evidence of an association between the risk alleles of SNPs on chromosome 9p and conventional risk factors such as lipid parameters or blood pressure levels, ${ }^{2,6}$ As stressed by the National Cancer Institute-National Human Genome Research Institute (NCINHGRI) Working Group on Replication in Association Studies, validation of the postulated gene-disease associations in a population different from that of the initial study is of great value. Thus, the aim of the present study was to examine whether the previously reported CAD-associated SNPs, including those located on chromosome 9p21, confer 
Table 1 Clinical Characteristics of the Control and MI Groups

\begin{tabular}{lccc}
\hline \hline & Controls & MI cases & $p$ value \\
\hline$n(M / F)$ & $2,475(1,102 / 1,373)$ & $589(510 / 79)$ & $<0.0001$ \\
Age $($ years $)$ & $65.1 \pm 11.0$ & $61.3 \pm 10.3$ & $<0.0001$ \\
BMI $\left(\mathrm{kg} / \mathrm{m}^{2}\right)$ & $22.8 \pm 3.1$ & $23.7 \pm 3.0$ & $<0.0001$ \\
$\%$ current smokers & 16.3 & 59.9 & 0.026 \\
$\%$ current drinkers & 45.4 & 40.3 & $<0.0001$ \\
$\%$ subjects with hypertension & 36.8 & 55.1 & $<0.0001$ \\
$\%$ subjects with diabetes & 9.3 & 42.0 & 0.10 \\
\hline subjects with hyperlipidemia & 52.0 & 55.7 & \\
\hline
\end{tabular}

Age and BMI are presented as mean \pm standard deviation; other variables are shown as percentage.

MI, myocardial infarction; BMI, body mass index.

susceptibility to MI in a Japanese population and to investigate the effect of these SNPs on cardiovascular risk factors.

\section{Methods}

\section{Study Population}

The inclusion criteria and study design of the Suita Study have been described previously9-15 In brief, the sample consisted of 14,200 men and women (30-79 years of age at enrollment), stratified by gender and 10-year age groups (10 groups and 1,420 subjects in each group) who had been randomly selected from the municipal population registry. They were all invited, by letter, to attend regular cycles of follow-up examination (every 2 years). Subjects who were recruited into the Suita Study between April 2002 and February 2004 and were free from CAD served as controls $(n=2,475)$. MI cases $(n=589)$ were randomly selected inand outpatients with documented MI who were enrolled in the Division of Cardiology at the National Cardiovascular Center between May 2001 and April 2003. Both controls and MI cases were of the same ethnicity (Japanese) and from the same geographical area. Only those who gave written informed consent were included in the study. The study protocol was approved by the Institutional Ethics Committee and the Committee on Genetic Analysis and Gene Therapy of the National Cardiovascular Center. Subjects with systolic blood pressure (SBP) $\geq 140 \mathrm{mmHg}$, diastolic blood pressure (DBP) $\geq 90 \mathrm{mmHg}$ and/or the current use of antihypertensive medication were categorized as having hypertension. Subjects who had total cholesterol (TC) $\geq 220 \mathrm{mg} / \mathrm{dl}$ or triglycerides $(\mathrm{TG}) \geq 150 \mathrm{mg} / \mathrm{dl}$, or were taking antihyperlipidemic medication, were classified as hyperlipidemic. The definition of type 2 diabetes were based on fasting blood glucose levels $\geq 126 \mathrm{mg} / \mathrm{dl}$, hemoglobin $(\mathrm{Hb})$ Alc $\geq 6.5 \%$, and/or current treatment for diabetes. Mean and maximum intima-media thicknesses (IMT) of the common carotid artery were determined by high-resolution ultrasonography?

\section{Genotyping Assays}

Ten SNPs were selected based on the results of genomewide association analyses: 7 SNPs (rs2943634, rs6922269, rs1333049, rs599839, rs17465637, rs501120 and rs17228212) from the WTCCC and German MI Family Study, 2 chromosome 9p21 SNPs (rs10757274 and rs2383206) identified by McPherson et $\mathrm{al}^{2}$ and $\mathrm{rs} 10757278$, the most significant SNP on chromosome 9p21 reported by Helgadottir et al! The sequences encompassing the selected SNPs were determined by an ABI 3730 Sequencer (Applied Biosystems, Foster City, CA, USA) in a subgroup of control subjects ( $\mathrm{n}=$ 192) for the assessment of minor allele frequency and the degree of linkage disequilibrium (LD). TaqMan allelic discrimination assays were performed on a 7900 HT (Applied Biosystems) according to the manufacturer's instructions. Genotype frequency distributions for each SNP were tested for agreement with Hardy-Weinberg equilibrium by SNPAlyze Ver.5.0.4 Pro (Dynacom, Chiba, Japan). The degree of LD among polymorphisms was evaluated using SNPAlyze Ver.5.0.4 Pro (Dynacom).

\section{Statistical Analysis}

Data are expressed as mean \pm standard deviation. Allele and genotype frequencies between control and MI subjects were compared by chi-square $\left(\chi^{2}\right)$ test. Odds ratio (OR) and 95\% confidence interval (CI) for the risk allele were estimated by logistic regression analysis with adjustment for age, sex, hypertension, diabetes, and current drinking and smoking habits. Continuous variables were tested for normality of distribution, and logarithmic transformation was applied for those with skewed distributions. Residuals, defined as the observed value minus predicted value, on the basis of confounding factors, were used for the phenotypegenotype association analysis by 1-way analysis of variance tests. Covariates included in the model were derived from multiple logistic regression analysis and used to calculate a residual value for each variable. The level of significance was adjusted for multiple testing by Bonferroni correction. Statistical analysis was performed using the JMP statistical package 7.0 (SAS Institute, Cary, NC, USA).

\section{Results}

Clinical characteristics of the controls and MI cases included for genotyping are summarized in Table 1. The proportion of male subjects was higher in the MI group than in the control group. Subjects with MI were younger and had a higher prevalence of smoking habit, diabetes and hypertension compared with controls $(\mathrm{p}<0.0001)$.

Among the 10 SNPs selected for the analysis, 4 that had minor allele frequency of less than 0.15 (rs2943634, rs6922269, rs599839 and rs17228212) were excluded. The 4 SNPs on chromosome 9p21 were highly correlated with each other. For simplicity, we present our results with a particular focus on rs1333049, which was in strong LD with $\mathrm{rs} 10757278\left(\mathrm{r}^{2}=0.98\right), \operatorname{rs} 2383206\left(\mathrm{r}^{2}=0.87\right)$ and rs10757274 $\left(\mathrm{r}^{2}=0.83\right)$.

Table 2 shows the genotype and risk allele frequencies for the 3 SNPs genotyped in 2,475 controls and $589 \mathrm{MI}$ subjects. OR for MI with the risk allele at rs1333049, rs17465637 and rs501120 estimated by logistic regression analyses with adjustment for age, sex, diabetes, hypertension and smoking habit were 1.47 (95\% CI, 1.15-1.89; 
Table 2 Logistic Regression Analysis of MI

\begin{tabular}{|c|c|c|c|c|c|c|c|c|c|c|c|c|c|c|c|}
\hline \multirow{3}{*}{ SNPS } & \multirow{3}{*}{ Chr } & \multirow{3}{*}{$\begin{array}{l}\text { Risk } \\
\text { allele }\end{array}$} & \multicolumn{2}{|c|}{ Risk allele frequency } & \multicolumn{6}{|c|}{ Genotype frequency } & \multirow{2}{*}{\multicolumn{2}{|c|}{$H W E^{*}$}} & \multirow{3}{*}{$\begin{array}{l}\text { OR for risk allele } \\
(95 \% \mathrm{CI})\end{array}$} & \multirow{3}{*}{$p$ value } & \multirow{3}{*}{$p$ value } \\
\hline & & & \multirow{2}{*}{$\begin{array}{c}\text { Control } \\
(n=2,475)\end{array}$} & \multirow{2}{*}{$\begin{array}{c}\text { MI cases } \\
(n=589)\end{array}$} & \multicolumn{3}{|c|}{ Control } & \multicolumn{3}{|c|}{ MI cases } & & & & & \\
\hline & & & & & $O O$ & $O X$ & $X X$ & $O O$ & $O X$ & $X X$ & control & MH cases & & & \\
\hline rs 1333049 & 9 & $C$ & 0.491 & 0.528 & 636 & 1,204 & 592 & 137 & 279 & 170 & 0.67 & 0.32 & $1.47(1.15-1.89)$ & 0.002 & 0.006 \\
\hline$r s 17465637$ & 1 & $C$ & 0.538 & 0.579 & 526 & 1,196 & 711 & 112 & 263 & 204 & 0.61 & 0.12 & $1.45(1.15-1.83)$ & 0.002 & 0.006 \\
\hline rs501120 & 10 & $T$ & 0.640 & 0.668 & 287 & 1,180 & 967 & 62 & 262 & 258 & 0.12 & 0.78 & $1.22(0.98-1.53)$ & 0.079 & 0.237 \\
\hline
\end{tabular}

Genotype frequencies are given for $O O, O X$ and $X X$ of the risk allele.

*Deviation from HWE was assessed by chi-square test, and $p$ values for controls and MI cases are shown.

${ }^{\dagger}$ All 3 SNPs were included in the logistic regression analyses, with adjustment for age, sex, diabetes, hypertension and smokers. BMI and the presence of hyperlipidemia were not significant predictors for MI and not included in the model.

${ }_{\sharp}$ Bonferroni correction for multiple testing was applied by multiplying the p value by 3 (the number of SNPs studied for association with MI).

SNP, single nucleotide polymorphism; Chr, chromosome; OO, non-carriers; OX, heterozygous carriers; XX, homozygous carriers; HWE, Hardy-Weinberg

Equilibrium; OR, odds ratio; CI, confidence interval. Other abbreviations see in Table 1.

Table 3 Association Analyses of rs1333049 and rs17465637 With Anthropometric and Metabolic Parameters in 2,475 Subjects

\begin{tabular}{|c|c|c|c|c|c|c|c|}
\hline \multirow{5}{*}{$n(M / F)$} & \multicolumn{3}{|c|}{ rs1333049 } & \multicolumn{3}{|c|}{$r s 17465637$} & \multirow{5}{*}{ Covariates } \\
\hline & $G G$ & 636 & $(292 / 344)$ & $C C$ & 711 & $(317 / 394)$ & \\
\hline & $G C$ & 1,204 & $(553 / 651)$ & $C A$ & 1,196 & $(529 / 667)$ & \\
\hline & $C C$ & 592 & $(244 / 348)$ & $A A$ & 526 & $(241 / 285)$ & \\
\hline & Genotype & $C C v$ & $C C+G C v$ & Genotype & $C C v$ & $C C+C A v$ & \\
\hline & & & & & & & \\
\hline Age (year) & 0.81 & 0.56 & 0.96 & 0.81 & 0.88 & 0.52 & \\
\hline Res. BMI $\left(\mathrm{kg} / \mathrm{m}^{2}\right)$ & 0.35 & 0.2 & 0.28 & 0.1 & 0.11 & 0.41 & Sex \\
\hline Res. mean IMT (mm) & 0.29 & 0.52 & 0.12 & 0.77 & 0.82 & 0.57 & Age, sex, BMI, SBP \\
\hline Res. maximum IMT (mm) & 0.78 & 0.73 & 0.65 & 0.19 & 0.07 & 0.52 & Age, sex \\
\hline Res. hs-CRP $(\mathrm{mg} / \mathrm{dl})^{a}$ & 0.79 & 0.49 & 0.85 & 0.07 & 0.05 & 0.63 & Age, BMI \\
\hline Res. $T C(\mathrm{mg} / \mathrm{dl})^{*}$ & 0.17 & 0.07 & 0.3 & 0.58 & 0.84 & 0.3 & Sex, WHR \\
\hline Res. $T G(m g / d l)^{a, *}$ & 0.90 & 0.98 & 0.65 & 0.56 & 0.29 & 0.59 & Sex, BMI, WHR, cigarette consumption \\
\hline Res. $H D L-C(m g / d l) *$ & 0.63 & 0.36 & 0.97 & 0.62 & 0.93 & 0.37 & Sex, BMI, WHR, cigarette and alcohol consumption \\
\hline Res. SBP $(m m H g) * *$ & 0.39 & 0.19 & 0.95 & 0.91 & 0.99 & 0.68 & Age, BMI, cigarette and alcohol consumption \\
\hline Res. $\mathrm{DBP}(\mathrm{mmHg}) * *$ & 0.37 & 0.19 & 0.93 & 0.31 & 0.25 & 0.59 & Age $^{2}, B M I$, alcohol consumption \\
\hline Res. glucose $(\mathrm{mg} / \mathrm{dl}) * * *$ & 0.85 & 0.93 & 0.62 & 0.5 & 0.8 & 0.31 & Sex, BMI, WHR \\
\hline Res. $H b A l c(\%) * * *$ & 0.92 & 0.83 & 0.8 & 0.83 & 0.59 & 0.64 & Age, BMI, cigarette consumption \\
\hline
\end{tabular}

$p$ values by analysis of variance test.

${ }^{a}$ Log-transformed values were used for the analysis. *Subjects taking anti-hyperlipidemic medication $(n=364)$ were excluded. **Subjects who were on medication for hypertension $(n=609)$ were excluded. ***Subjects being treated for diabetes $(n=113)$ were excluded.

Covariates included for the calculation of residual values are shown. Alcohol consumption was expressed as ethanol ( $g$ ) per day. Cigarette consumption was defined as the product of the number of years of smoking and the number of cigarettes consumed per day.

Res, residual; IMT, intima-media thickness; SBP, systolic blood pressure; hs-CRP, high-sensitivity C-reactive protein; TC, total cholesterol; TG, triglycerides; WHR, waist to hip ratio; HDL-C, high-density lipoprotein-cholesterol; DBP, diastolic blood pressure; HbAlc, hemoglobin Alc. Other abbreviation see in Table 1.

nominal $\mathrm{p}=0.002$; corrected $\mathrm{p}=0.006), 1.45$ (95\% CI, 1.15 1.83 ; nominal $\mathrm{p}=0.002$; corrected $\mathrm{p}=0.006)$ and 1.22 (95\% CI, 0.98-1.53; nominal $\mathrm{p}=0.079$; corrected $\mathrm{p}=0.237$ ), respectively. The association of rs1333049 and rs17465637 with MI remained significant after adjustment for multiple testing. Inclusion of the 2 positive SNPs (rs1333049 and rs 17465637) instead of all 3 SNPs in the logistic regression analysis did not significantly alter the results; ORs adjusted for age, sex, diabetes, hypertension and smoking habit were 1.46 for rs 1333049 ( $95 \%$ CI, $1.14-1.87$; nominal $\mathrm{p}=0.0027$; corrected $\mathrm{p}=0.005)$ and 1.44 for rs 17465637 (95\% CI, 1.14 1.82; nominal $\mathrm{p}=0.0024$; corrected $\mathrm{p}=0.005$ ), respectively. The interaction terms (rs1333049*rs17465637, rs1333049* age, rs17465637*age, rs 1333049*sex, rs17465637*sex, rs 1333049*BMI, rs17465637*BMI) did not reveal any significant association with MI after taking diabetes, hypertension and smoking habit into consideration.

Table 3 shows the association analysis of the 2 positive SNPs (rs1333049 and rs 17465637) with anthropometric and metabolic parameters in the 2,475 controls. Confounding factors included in the model to calculate a residual value for each variable are detailed in Table 3 . Irrespective of whether the comparison was made between the genotype groups under the additive genetic model, between homozygous carriers of the risk allele and the combined group of non-carriers and heterozygotes or between those with at least one risk allele and those without it, genetic variants at rs1333049 and rs17465637 were not associated with differences in age, body mass index (BMI), mean and maximal IMT, high-sensitivity C-reactive protein (hs-CRP), TC, TG, high-density lipoprotein-cholesterol (HDL-C), SBP, DBP, fasting glucose, or $\mathrm{Hb} \mathrm{A}_{1 \mathrm{c}}$ in this Japanese population.

\section{Discussion}

The association of the 2 SNPs (rs1333049 and rs17465637) with MI was reproduced in this Japanese sample, yielding an adjusted OR of 1.47 (95\% CI, 1.15-1.89) and 1.45 (95\% CI, 1.15-1.83) for rs1333049 and rs17465637, respectively. The risk allele frequencies for rs1333049 observed in our study sample ( 0.53 among cases and 0.49 among controls) were similar to those reported previously in the WTCCC 
study (0.55 among cases and 0.47 among controls) and the German MI Family Study (0.54 among cases and 0.48 among controls). During the preparation of this article, a report investigating the association between rs1333049 and CAD in a Japanese population was published and replicated the association. 16 The risk allele frequency reported in that study was similar to ours: 0.49 for controls $(n=1,151)$ and 0.55 for patients with CAD $(n=604)$. Our results, taken together with data from that study, confirm that the previously reported CAD-associated SNP on chromosome 9p21 (rs1333049) confers susceptibility to MI in Japanese.

The risk allele frequency of SNP rs17465637 was slightly lower in our study sample ( 0.54 among controls and 0.58 among cases) than in populations of European ancestry; $0.71-0.74$ in controls and $0.75-0.76$ in CAD/MI cases. This observation was comparable to the difference found in the $\mathrm{C}$ allele frequency between the HapMap Japanese (JPT, 0.556) and CEU (CEPH European ancestry, 0.750) samples! ${ }^{17}$

SNP rs501120 at the $10 \mathrm{q} 11$ locus (nearby gene, LOC728347) did not show any association with MI in this Japanese sample. The risk allele frequency in the controls (0.640) was almost identical to that from the HapMap JPT data (0.636), but lower than that reported in controls of white European origin (0.84-0.87).

We attempted to investigate the effect of the 2 positive SNPs (rs1333049 and rs17465637) on anthropometric and metabolic parameters in Japanese subjects without MI. None of the genotypes of rs1333049 and rs17465637 was associated with conventional risk factors such as BMI, plasma lipid profile, blood pressures, glucose, or Hb A1c. Homozygosity for the risk allele of rs1333049 and rs17465637 also had no effect on circulating levels of hs-CRP, an inflammatory marker that has been shown to be associated with cardiovascular events ${ }^{18-20}$ Carotid IMT has been used as a non-invasive measure of atherosclerosis ${ }^{21-23}$ In our present analysis, IMT of the common carotid artery assessed by high-resolution ultrasonography was not associated with the risk allele of rs 1333049 or rs 17465637.

The lack of an association between the genotypes of rs1333049 and the anthropometric and metabolic parameters observed in our study is in accordance with previous studies showing no effect of the chromosome 9p21 susceptibility locus on cardiovascular risk factors?2,6 Results from the Atherosclerosis Risk in Communities Study (ARIC) and Copenhagen City Heart Study (CCHS) demonstrated no significant associations between the genotypes of CADassociated SNPs (rs10757274 and rs2383206) and the risk factors including BMI, glucose, TC, TG, low-density lipoprotein-cholesterol, HDL-C, SBP, DBP and hs-CRP? Although the chromosome 9p21 region has emerged as a susceptibility locus for CAD, showing consistent associations across different populations, the increased risk associated with genetic variability at this locus appears not to be accounted for by the established risk factors. In the aforementioned replication study conducted in Japanese ${ }^{16}$ there was no association between the severity of coronary atherosclerosis and the prevalence of the risk allele of rs1333049.

It remains to be determined whether nearby genes, such as $C D K N 2 A, C D K N 2 B$ or $M T A P$, are responsible for the pathogenesis of CAD or if unknown mechanisms are involved. Although a large anti-sense non-coding RNA has been suggested as a candidate susceptibility gene for this locus, 6 only limited data are available to date.

The association between rs17465637 and MI was reproduced in the present Japanese sample. Although many studies have focused on the CAD-associated SNPs on chromosome 9p21, an intronic SNP of rs17465637 in the melanoma inhibitory activity family, member 3 (MIA3) gene on chromosome $1 \mathrm{q} 41$ has received little attention. MIA3, also referred to as $A R N T$ or $T A N G O$, belongs to the melanoma inhibitory activity gene family and may have a role in tumor suppression.24,25 As with the case of rs1333049, the classic risk factors appear not to be related to the increased MI risks associated with rs 17465637.

In the present study, significant differences in age, sex, BMI, smoking habit, hypertension and diabetes were noted between the MI and control groups, which often occurs in case-control studies. We attempted to minimize the potential effects of background differences with the multiple logistic regression analysis in which several confounding factors were included. However, there is the possibility that an inability to adjust for unknown or undetermined confounders could lead to a spurious or false-negative association. Moreover, in a cross-sectional case-control study design, only survivors from MI would be included in the case group. Thus, a large-scale prospective cohort study will be necessary to confirm that the genetic variants on chromosome 9p21 and/or 1q41 predispose to susceptibility to MI. However, with the accumulating results from many validation studies with positive associations, it is highly likely that the observed associations in this Japanese sample are valid.

Given the lack of associations between the CAD-associated SNPs and classic risk factors, further investigations focusing on the underlying mechanisms will provide new insight into the pathophysiology of cardiovascular disease, leading to improved prevention and treatment strategies.

\section{Acknowledgement}

We would like to thank all those who participated in the study. In addition, we gratefully acknowledge all the members of Suita City Health Center and the Suita Medical Association.

The present study was supported by a research grant from the Program for the Promotion of Fundamental Studies in Health Sciences of the National Institute of Biomedical Innovation, Japan.

\section{References}

1. Helgadottir A, Thorleifsson G, Manolescu A, Gretarsdottir S, Blondal $\mathrm{T}$, Jonasdottir A, et al. A common variant on chromosome 9p21 affects the risk of myocardial infarction. Science 2007; 316: 1491 1493.

2. McPherson R, Pertsemlidis A, Kavaslar N, Stewart A, Roberts R, Cox DR, et al. A common allele on chromosome 9 associated with coronary heart disease. Science 2007; 316: 1488-1491.

3. The Wellcome Trust Case Control Consortium. Genome-wide association study of 14,000 cases of seven common diseases and 3,000 shared controls. Nature 2007; 447: 661-678.

4. Samani NJ, Erdmann J, Hall AS, Hengstenberg C, Mangino M, Mayer B, et al. Genome-wide association analysis of coronary artery disease. N Engl J Med 2007; 357: 443-453.

5. Shen GQ, Li L, Rao S, Abdullah KG, Ban JM, Lee BS, et al. Four SNPs on chromosome 9p21 in a South Korean population implicate a genetic locus that confers high cross-race risk for development of coronary artery disease. Arterioscler Thromb Vasc Biol 2008; 28: $360-365$.

6. Broadbent HM, Peden JF, Lorkowski S, Goel A, Ongen H, Green F, et al. Susceptibility to coronary artery disease and diabetes is encoded by distinct, tightly linked, SNPs in the ANRIL locus on chromosome 9p. Hum Mol Genet 2008; 17: 806-814.

7. Shen GQ, Rao S, Martinelli N, Li L, Olivieri O, Corrocher R, et al. Association between four SNPs on chromosome 9p21 and myocardial infarction is replicated in an Italian population. J Hum Genet 2008; 53: 144-150.

8. Chanock SJ, Manolio T, Boehnke M, Boerwinkle E, Hunter DJ, Thomas G, et al. Replicating genotype-phenotype associations. 
Nature 2007; 447: 655-660.

9. Mannami T, Konishi M, Baba S, Nishi N, Terao A. Prevalence of asymptomatic carotid atherosclerotic lesions detected by high-resolution ultrasonography and its relation to cardiovascular risk factors in the general population of a Japanese city: The Suita study. Stroke 1997; 28: $518-525$.

10. Iwai N, Katsuya T, Mannami T, Higaki J, Ogihara T, Kokame K, et al. Association between $S A H$, an acyl-CoA synthetase gene, and hypertriglyceridemia, obesity, and hypertension. Circulation 2002; 105: $41-47$.

11. Shioji K, Kokubo Y, Mannami T, Inamoto N, Morisaki H, Mino Y, et al. Association between hypertension and the alpha-adducin, beta1-adrenoreceptor, and G-protein beta3 subunit genes in the Japanese population: The Suita study. Hypertens Res 2004; 27: $31-$ 37.

12. Kokubo Y, Iwai N, Tago N, Inamoto N, Okayama A, Yamawaki H, et al. Association analysis between hypertension and $C Y B A, C L C N K B$, and $K C N M B 1$ functional polymorphisms in the Japanese population: The Suita Study. Circ J 2005; 69: 138-142.

13. Iwai N, Kajimoto K, Kokubo Y, Okayama A, Miyazaki S, Nonogi H, et al. Assessment of genetic effects of polymorphisms in the MCP-1 gene on serum MCP-1 levels and myocardial infarction in Japanese. Circ J 2006; 70: 805-809.

14. Iwai N, Kajimoto K, Tomoike H, Takashima N. Polymorphism of CYP11B2 determines salt sensitivity in Japanese. Hypertension 2007; 49: 825-831.

15. Takashima N, Shioji K, Kokubo Y, Okayama A, Goto Y, Nonogi H, et al. Validation of the association between the gene encoding proteasome subunit alpha type 6 and myocardial infarction in a Japanese population. Circ J 2007; 71: 495-498.

16. Hinohara K, Nakajima T, Takahashi M, Hohda S, Sasaoka T, Nakahara K, et al. Replication of the association between a chromo- some 9p21 polymorphism and coronary artery disease in Japanese and Korean populations. J Hum Genet 2008; 53: 357-359.

17. The International HapMap Consortium. A haplotype map of the human genome. Nature 2005; 437: 1299-1320.

18. Blake GJ, Rifai N, Buring JE, Ridker PM. Blood pressure, C-reactive protein, and risk of future cardiovascular events. Circulation 2003; 108: 2993-2999.

19. Koenig W, Lowel H, Baumert J, Meisinger C. C-reactive protein modulates risk prediction based on the Framingham Score: Implications for future risk assessment: Results from a large cohort study in southern Germany. Circulation 2004; 109: 1349-1353.

20. Ridker PM, Buring JE, Cook NR, Rifai N. C-reactive protein, the metabolic syndrome, and risk of incident cardiovascular events: An 8-year follow-up of 14719 initially healthy American women. Circulation 2003; 107: 391-397.

21. Lorenz MW, von Kegler S, Steinmetz H, Markus HS, Sitzer M. Carotid intima-media thickening indicates a higher vascular risk across a wide age range: Prospective data from the Carotid Atherosclerosis Progression Study (CAPS). Stroke 2006; 37: 87-92.

22. Lorenz MW, Markus HS, Bots ML, Rosvall M, Sitzer M. Prediction of clinical cardiovascular events with carotid intima-media thickness: A systematic review and meta-analysis. Circulation 2007; 115: $459-$ 467.

23. O'Leary DH, Polak JF, Kronmal RA, Manolio TA, Burke GL, Wolfson SK Jr. Carotid-artery intima and media thickness as a risk factor for myocardial infarction and stroke in older adults: Cardiovascular Health Study Collaborative Research Group. $N$ Engl J Med 1999; 340: 14-22.

24. Arndt S, Bosserhoff AK. TANGO is a tumor suppressor of malignant melanoma. Int J Cancer 2006; 119: 2812-2820.

25. Arndt $\mathrm{S}$, Bosserhoff AK. Reduced expression of TANGO in colon and hepatocellular carcinomas. Oncol Rep 2007; 18: 885-891. 